Original article

\title{
Evaluating the effect of a fish oil supplement on the Omega-3 Index of three professional cyclists competing in the Tour de France: a case study
}

\author{
Michael J. Macartney ${ }^{1,2}$, Marcel Hesseling 3, Raquel Ortolano 3, Peter L. McLennan 1,2, Gregory E. \\ Peoples ${ }^{1,2}$ \\ Graduate Medicine, School of Medicine, University of Wollongong, Wollongong, Australia. \\ Centre for Medical and Exercise Physiology, Faculty of Science Medicine and Health, University of \\ Wollongong, Wollongong, Australia. \\ 3 Astana Premier-Tech Team, Nutritional and Medical Staff.
}

* Correspondence: Gregory E. Peoples. peoples@uow.edu.au

Received: 18 January 2021; Accepted: 27 June 2021; Published: 30 June 2021

\begin{abstract}
Modifying the Omega-3 Index (O3I) in the elite endurance cyclist is desirable for cardiovascular and anti-inflammatory effects. This case study evaluated the effect of evidencebased fish oil supplementation, providing long chain omega-3 eicosapentaenoic acid (EPA) and docosahexaenoic acid (DHA), on three elite riders taking part in the Tour de France (TdF). The O3I of three riders was evaluated using a finger prick blood sample at the start of the season. Riders were then advised to consume daily fish oil supplements (NAMEDSPORT, Italy) according to their initial O3I to achieve $>8 \%$ membrane EPA+DHA. Follow up blood samples were collected before and after the TdF. At baseline, the O3I of rider one (R1: 6.23\%) and two (R2: $6.77 \%)$ were categorised as sub-optimal $(<8 \%)$ whereas rider three (R3: $9.66 \%)$ was $>8 \%$. Two daily fish oil capsules (1118 mg EPA/ $458 \mathrm{mg}$ DHA) increased the O3I to in both R1 (8.40\%) and R2 $(9.69 \%)$ by the start of the TdF and this was maintained upon completion of the race (R1: $8.81 \%$; R2: $9.37 \%)$. The O3I of R3 (9.24\%) was maintained by consuming one daily fish oil capsule (559 mg EPA/229 mg DHA) in the lead up to the TdF and upon completion their O3I was similar $(9.52 \%)$ despite increasing their intake to two daily capsules during the TdF. This case study demonstrates that an elite cyclist, consuming 1-2 daily fish oil capsules, can achieve and maintain a high O3I, in the favour of cardio-protection and anti-inflammation during arduous competition.
\end{abstract}

Keywords: Elite cycling; Grand tour; Omega-3 Index; fish oil; EPA; DHA.

\section{Introduction}

Professional cycling is physiologically demanding and this is characterised by the Grand Tours. The Tour de France (TdF) involves the teams riding in excess of 3000 $\mathrm{km}$ with average speeds approaching 40 $\mathrm{km} /$ hour (Santalla et al. 2012). The combination of flat, undulating and mountain stage interspersed with time trial stage necessitates continuous and protracted endurance activity, combined with intermittent periods of explosive action (Fernández-García et al. 2000). Accordingly, the physiological characteristic of the professional road cyclist is an exceptional combination of high aerobic capacity and ability to sustain close to maximal aerobic power (Novak and Dascombe 2014).

To support such requirements, nutritional strategies, such as acute and chronic intake of carbohydrate and protein, must be carefully executed to enhance qualities of endurance and recovery to obtain

() 2021 fist author, licensee JSC. This is an Open Access article distributed under the terms of the Creative Commons Attribution License ((http://creativecommons.org/licenses/by/4.0/) which permits unrestricted use, distribution, and reproduction in any medium, provided 
performance success at the elite level (Burke 2001). The optimal provision of macronutrients consisting of $65 \%$ carbohydrate and $20 \%$ protein leaves approximately $15 \%$ of the composition from fat. The profile of fat intake is intriguing, in that as well as providing a metabolic energy source, the relative proportions of saturated, monounsaturated and polyunsaturated fatty acids differentially modify cell membranes of tissues, such as skeletal muscle (Andersson et al. 2002) that includes physiological adaptation (Helge et al. 2001).

The typical Western style diet is less than optimal in providing long chain omega3 polyunsaturated fatty acids (LCn-3PUFA) (Micha et al. 2014). This can also be implied from global erythrocyte eicosapentaenoic acid (EPA) and docosahexaenoic acid (DHA) concentrations (Stark et al. 2016), referred to as the Omega-3 Index (O3I: EPA+DHA\%), with an optimal target $>8 \%$ for heart function (Harris and Von Schacky 2004). In a cohort of 106 German elite winter-endurance athletes, consuming their usual training diet, the mean O3I was reported to be $<5 \%$ and only one individual was $>8 \%$ (von Schacky et al. 2014). Accordingly, supplementation of fish oil is likely required in many athletes to achieve an optimal O3I.

It is well-established that regular dietary consumption of fish oils leads to the preferential incorporation of EPA+DHA into myocardial and skeletal muscle membrane phospholipids, via replacing omega-6 arachidonic acid (AA), in a dose-related manner (Slee et al. 2010), and according to fibre type (Macartney et al. 2019). Of physiological consequence, a number of actions have been established (particularly when the system is under stress); these include reduced cardiovascular demands, alongside increased membrane O3I (Peoples et al. 2008), enhanced anti-inflammatory pathways (alongside a lower whole blood $\mathrm{AA} / \mathrm{EPA}$ ratio) and pro-resolution actions (alongside a lower membrane omega6/omega-3 ratio). Therefore, the avid incorporation of LCn-3PUFA into membranes of tissues important to cycling performance (cardiac and skeletal muscle), in combination with their physiological actions, highlights the opportunity of optimising specific dietary fatty acid consumption protocols and advice for athletes whilst not interfering with the performance and recovery objectives via optimal intakes of carbohydrate and protein (Burke 2001).

Contrary to the common nutritional goals for high carbohydrate and protein intake in preparation for and during competition, athletes are scarcely provided advice for fatty acid intake regimes, and definitely not informed from blood biomarker sampling. This case study aimed to evaluate the fatty acid profile of three elite riders during the early cycling calendar, then provide fish oil supplement advice, to these riders, according to evidence-based dose response of LCn-3PUFA (Slee et al. 2010) to achieve an optimal O3I $(>8 \%)$ and reduce the omega-6/omega-3 $(<5)$ and AA/EPA $(<11)$ ratios (collectively referred to as 'omega-3 status' from this point forward) over the course of the physically demanding TdF.

\section{Materials and Methods}

Participants and ethical approval.

Each participant was a professional rider (European), contracted to the same Union Cycliste Internationale (UCI; ranked within $50^{\text {th }}$ to $260^{\text {th }}$ place) registered cycling team, at the time of the case study (season 2020). Rider one and two each had eight years of professional cycling experience and rider three had 16 years of professional cycling experience (Table 1). The three riders, at the commencement of the case study, were anticipated to be part of TdF in terms of their respective role in the team (Table 1). The study procedures were approved by the University of Wollongong Human Research ethics committee and the study was conducted in accordance with the Declaration of Helsinki. The three cyclists provided written consent to the procedures and publication of their de-identified data. 
Table 1. Characteristics of the three professional riders as of the start of the 2020 professional cycling season.

\begin{tabular}{|l|c|c|c|}
\hline & Rider 1 & Rider 2 & Rider 3 \\
\hline Age (y) & 30 & 28 & 37 \\
\hline Height (cm) & 183 & 174 & 186 \\
\hline $\begin{array}{l}\text { Body mass } \\
(\mathrm{kg})\end{array}$ & 72 & 74 & 73 \\
\hline $\begin{array}{l}\text { Body mass } \\
\text { index }\end{array}$ & 21.5 & 24.5 & 21.1 \\
\hline $\begin{array}{l}\text { Professional } \\
\text { yrs }\end{array}$ & 8 & 8 & 16 \\
\hline Career wins & 6 & 26 & 42 \\
\hline Current role & Domestic & Climber & Puncheur \\
\hline
\end{tabular}

Study design.

This case study occurred during the cycling calendar (2020) in the lead up to and including the TdF. The European professional cycling season was delayed due to COVID-19 restrictions, and therefore the time points described are in line with the alterations to the commencement of the TdF. The months of July and August were dedicated to single or multi-stage races leading into the three Grand Tours (September - November). The whole-blood fatty acid profile of each rider was objectively measured in the first week of July, the first week of September (commencement of TdF) and on the morning of the final stage (Paris). The membrane fatty acid composition in the first week of July was used to inform the supplemental requirement to complement the provision of LCn-3PUFA obtained from the rider's training and competition diet.

\section{Whole blood fatty acid profile.}

Each rider provided a morning fasted and adequately hydrated, blood sample using the finger prick method (conducted according to best practice guidelines by RO). The drop of blood was spotted onto the commercially available collection card for independent analysis (Omega-Quant, South Dakota, United States). The sample card was immediately sent to Omega-Quant (USA) to determine the whole-blood fatty acid profile using state of the art gas chromatography. Each fatty acid in the whole blood is individually identified using high quality standards and then described as a relative percentage (\%) of all the fatty acids. The red blood cell O3I, a marker of cardiac and skeletal muscle membrane incorporation, was then calculated according to a validated algorithm $(\mathrm{r}=0.96)$ (Harris and Polreis 2016).

\section{Description of nutritional objectives.}

Each rider followed their personalised diet plan based on the guidelines and calculations provided by the team nutritionist $(\mathrm{MH})$ in accordance with the sport nutrition guidelines (Kerksick et al. 2017). The objective, especially during the $\mathrm{TdF}$, was to maintain their bodyweight during training and racing. Everyday riders received guidelines on how to compose their meals so that the total energy requirements (MJ) and macronutrient intakes were matched to optimise performance and recovery, especially between each stage. On race days riders consumed five meals (breakfast, post-race, arrival at hotel, dinner and pre sleep), prepared by the team Chef, and received advice on what to eat on the bike, which was focussed on carbohydrates (30-90 g/h).

\section{Fish oil supplement and advice to the riders.}

The three riders had access to an approved omega-3 supplement (NAMEDSPORTS, Italy). The Omega-3 Double Plus Soft Gels (1 gram each) contained concentrated fish oil and tocopherol-rich extract (antioxidant), for the stability of the fatty acids, in a gelatine capsule (soft gel). Each capsule of LCn3PUFA rich fish oil contained EPA (559 mg) and DHA (229 mg).

On the basis of the first fatty acid profile (July), omega-3 supplementation advice was provided according to an established dose- 
response that optimises cell membrane incorporation of EPA+DHA. In summary, a minimum target of 500-600 mg of EPA+DHA per day optimises skeletal muscle (Macartney et al. 2019) and cardiac membrane concentrations (Slee et al. 2010), reflected by changes in red blood cell membranes over the course of eight weeks (Hingley et al. 2017).

In order to achieve an optimal omega- 3 status over the course of 6-8 weeks, before the scheduled start of the TdF, the first objective was to evaluate baseline LCn-3PUFA membrane concentrations. A rider identified with $\mathrm{O} 3 \mathrm{I}<8 \%$ was advised to consume $2 \mathrm{x}$ Omega-3 capsules (NAMEDSPORT, Italy) each day, providing a total of $1118 \mathrm{mg}$ of EPA and $458 \mathrm{mg}$ of DHA. A rider identified with O3I $>8 \%$ was advised to consume $1 \times$ Omega3 capsule (NAMEDSPORT, Italy) each day, providing $559 \mathrm{mg}$ EPA and $229 \mathrm{mg}$ DHA. The capsules were consumed with food as part of a mealtime and continued through the duration of TdF (September) to establish a maintained state.

\section{Results}

Omega-3 status evaluation.

The July sample represented the usual intake of dietary fatty acids including the LCn-3PUFA. Rider one self-reported 2-3 intakes of a fish product each week and a very low intake of LCn-3PUFA as omega-3 supplement (120 mg EPA and $70 \mathrm{mg}$ DHA) for 4-6 weeks, in the three months preceding the sample. Rider two self-reported 1-2 intakes of a fish product each week and no omega-3 supplement intake. Rider three also reported 1-2 intakes of a fish product each week and had consumed an omega-3 supplement providing $559 \mathrm{mg}$ of EPA and $229 \mathrm{mg}$ of DHA during the four weeks preceding the July sample.

Rider one recorded the highest sum saturated fatty acids (SFA) and monounsaturated fatty acids (MUFA) whereas rider two was highest for the sum of omega-6 fatty acids (Table 2). The sum of omega-3 fatty acids was highest in rider three compared to both others, primarily due to the elevated EPA+DHA concentrations, in line with the self-reported intake of an omega-3 supplement totalling $788 \mathrm{mg}$ per day. When expressed as ratios the omega-6/omega-3 and AA/EPA were lowest in rider three (Figure $1 \mathrm{~A}$ and $\mathrm{B})$. The O3I of rider three $(9.66 \%)$ was in the optimal range $(>8 \%)$, whereas rider one $(6.23 \%)$ and two $(6.76 \%)$ were $<8 \%$ and classified as sub-optimal (Figure 1C). Notably, the EPA+DHA contributions were not equivalent, where rider two had higher relative DHA contribution compared to rider one (Table 2, annexes).

Omega-3 status following fish oil supplementation advice.

Rider one: Two capsules per day (1118 mg EPA, 458 mg DHA) successfully increased the O3I to $8.4 \%$ by the first week of the Tour (sample two) (Figure 1C). This increase was underpinned by an increase of whole blood EPA+DHA (Table 2). Consequently, both omega-6/omega-3 and AA/EPA ratios decreased prior to the TdF (Figure $1 \mathrm{~A}$ and $\mathrm{B}$ ). By the final day of the tour (sample three), the omega-3 status was maintained and the indices unchanged from the start of the tour.

Rider two: Two capsules per day also successfully increased the O3I to an optimal $9.69 \%$ by the TdF (sample two) (Figure 1C). This increase was underpinned by a major contribution from EPA and less so from DHA (Table 2). As was the case for rider one, both omega-6/omega-3 and especially AA/EPA ratios were decreased by the commencement of the TdF and by the final day of the tour (sample three), the omega-3 status was maintained and the indices unchanged from the start of the tour (Figure 1).

Rider three: In contrast, rider three with an O3I in excess of $9.5 \%$ (Figure 1C), was initially advised to consume one capsule per day (559 mg EPA, 229 mg DHA) which guaranteed the minimum daily recommendation for total LCn-3PUFA intake (500-600 mg). This dose was maintained over the course of July and August and 
consequently the O3I was maintained over
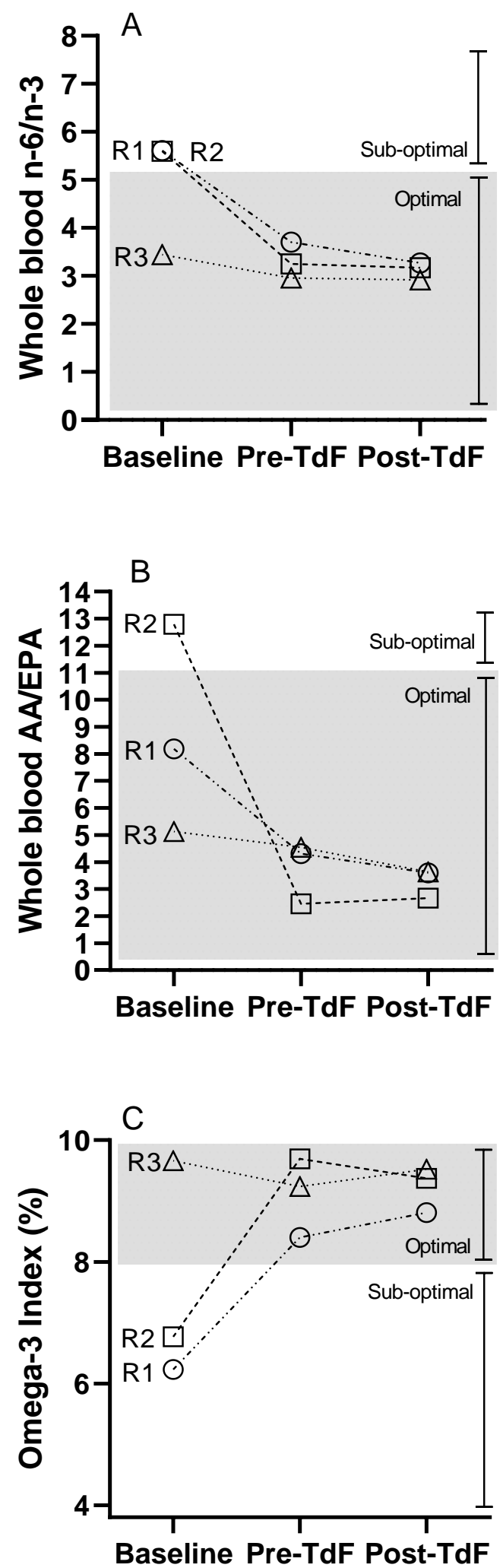

Figure 1Figure 1. T Baseline, pre-TdF and post TdF (A) whole blood $n-6 / n-3$ ratio $(B) A A / E P A$ ratio and (C) Omega-3 Index (\%) for the three riders (R1 O; R2: $\square$; $R 3 \Delta)$.
$9 \%$ whilst also further improving the already

well-established omega-6/omega-3 and AA/EPA ratios (Figure 1). In discussion with the team staff, the dose was increased from one to two capsules per day over the duration of the tour. The premise was to observe if doubling the LCn-3PUFA intake would modify the indices beyond that maintained on one capsule per day. Consequentially, by the final day of the tour (sample three), the omega-3 status was maintained and the indices unchanged from the start of the tour, despite double the dose (Figure 1).

\section{Discussion}

This unique case study evaluated the whole blood fatty acid profile of three professional UCI road cyclists in the early competitive cycling season. In response to this profiling, the omega-3 status was improved in two riders and optimally maintained in the third rider over the course of the TdF. These important observations demonstrate the efficacy of fish oil, providing both EPA+DHA, to be translated from the laboratory (Hingley et al. 2017) to the elite cyclist preparing and competing in a Grand Tour. Maintaining this optimal state of the O3I $>8 \%$ supports of optimal skeletal muscle incorporation of EPA and DHA (Macartney et al. 2019) cardiac protection (Macartney et al. 2020), anti-inflammation and proresolution (Calder et al. 2010), whilst achieving the personalised carbohydrate and protein nutritional goals for training, racing and recovery.

The self-reported intake of LCn-3PUFA, either as seafood, or the consistent use of a quality fish oil supplement matched the initial whole blood fatty acids profile of each rider. Rider three with optimal omega-3 status clearly attributable to the daily intake of close to $800 \mathrm{mg}$ of EPA+DHA as a supplement, in line with demonstrable doseresponse for cardiac and skeletal muscle (Macartney et al. 2019; Slee et al. 2010). Whereas, despite rider one and two reporting intakes of seafood several times per week, the lower and sub-optimal omega-3 status are 
likely attributable to the very low dose of LCn-3PUFA supplement (<200 mg per day) for rider one and the absence of a supplement for rider two. There is currently no comparative data in elite professional cyclists at this time. Although notably, both rider one and two had an O3I that was higher than the mean value $(4.97 \%)$ previously reported in the German elite winter endurance athletes (von Schacky et al. 2014). Nonetheless, some cautionary comparison can be made to broader higher performance athletic groups, such as a recent study that revealed that despite $39 \%$ of NCAA athletes consuming the recommended amount of dietary fish per week, only $6 \%$ met the requirement for EPA+DHA intake (Ritz et al. 2020). The same mean and distribution were recently reported among two other cohorts of American College Footballers (Anzalone et al. 2019; Drobnic et al. 2017).

As there was less than two months to the start of the TdF, rider one and two were advised to consume two capsules per day, a daily dose of $1118 \mathrm{mg}$ EPA + $458 \mathrm{mg}$ DHA, which is approximately double the recommended daily intake of LCn-3PUFA and achieves maximal membrane incorporation of EPA+DHA (Macartney et al. 2019; Slee et al. 2010) and demonstrated to achieve an O3I $(>8 \%)$ in laboratory studies (Hingley et al. 2017; Macartney et al. 2014). Most notably, this dose of two capsules per day was also effective in modifying the omega-3 whole blood profile (omega6/omega-3 $<5$ and AA/EPA $<11$ ), without interference from the background diet strategies for carbohydrate or protein and in accordance with each riders' specific needs, nor having to be concerned about the other specific fatty acids, such as the omega- 6 group. This last point is most relevant, as it is has been claimed that the 'background diet should be balanced for omega- 6 and omega3 fatty acids by lowering the omega- 6 oil' (Simopoulos 2007), where in fact, due to the preferential incorporation of EPA+DHA, simply providing the consistent $500-600 \mathrm{mg}$ per day of LCn-3PUFA (McLennan 2014) should be the target for all athletes including endurance cyclists to create an optimal omega-3 status.

Whilst enduring the significant demands of the TdF, all three riders consumed two capsules per day and there was a clear nadir achieved for omega-3 status, typified by the plateau in the O3I $>8 \%$. As rider three had also been increased from one to two capsules for the TdF, this provided further evidence that an optimal and established membrane LCn-3PUFA incorporation plateau can be effectively maintained alongside a circulating fatty acids profile favouring anti-inflammation (reduced AA/EPA) and pro-resolution (reduced omega-6/omega-3 ratio) activities. Acknowledging, the observations are limited to three elite cyclists completing the $\mathrm{TdF}$, the desired omega-3 status achieved via dietary supplementation are in direct contrast to previously reported effects of arduous exercise and training. For example, higher weekly running distance has been reported to be associated with a lower O3I and higher AA/EPA ratio (Davinelli et al. 2019). Unfortunately, although this study excluded those taking fish oil supplements, the habitual diet of participants was only considered for one week before the blood sample. Given the reported O3I of their sample, there is clear evidence for LCn3PUFA intake through food sources such as fish and seafood. In the same instance, the O3I of National Football League players decreased over the competition season (Blue et al. 2019 ) from values that were already sub-optimal. In summary, the more powerful influencer of the omega-3 status, being dietary intake of LCn-3PUFA, were largely un-accounted. To help resolve these contradictions future research should (i) expand to include riders both consuming and not consuming an omega-3 supplement from one or more professional cycling teams taking part in argueablly the most rigorus endurance competiton, and (ii) document the omega- 3 status of the riders over the long term, alongside LCn-3PUFA intake. 


\section{Practical Applications.}

Evaluating the O3I in the elite cyclist can improve individualise advice for the consumption of EPA and DHA. A rider with consistent intake of EPA and DHA (via fish and fish oil supplements) can sustain an optimal omega-3 status with as little as one fish oil capsule per day, providing a total of $800 \mathrm{mg}$ of EPA and DHA (just above the daily recommendation). A rider with a suboptimal O3I can be rectified over 1-2 months by providing approximately doubled that dose. Most importantly this optimal omega-3 status can be maintained with no impact on the nutritional plans and goals of the elite cyclist competing in the Tour de France, which include total energy requirements and the provision of carbohydrate and protein.

\section{Conclusions}

We have demonstrated that an achievable dose of quality fish oil, proven from laboratory studies, can rapidly rectify an elite rider's omega-3 status, independent to the nutritional objectives required during the TdF. Further, the optimised omega-3 status (O3I $>8 \%$, omega-6/omega-3 $<5$, AA/EPA <11) was maintainable within all three riders using these proven doses, without factoring in the overall fatty acid profile of the diet (that is, the omega-6 background). It remains to be determined if these observations can now be translated to full professional cycling teams, alongside promoting the physiological benefits, including cardiovascular, anti-inflammation and pro-resolution effects within the elite rider taking part in a Grand Tour.

\section{Supplementary Materials: None}

Funding: This research received no external funding.

Acknowledgments: We thank the three riders for their provision of data.

Conflicts of Interest: The authors declare no conflict of interest.

Authorship: The study was designed by GEP, MH and RO; data were collected by $\mathrm{MH}$ and RO; data analysis and interpretation by GEP, PMcL, MM and manuscript preparation were undertaken by
GEP, MM, MH, RO, PMcL. All authors approved the final version of the paper. The authors declare no conflict of interest.

\section{References}

1. Andersson A, Nalsen C, Tengblad S et al. (2002) Fatty acid composition of skeletal muscle reflects dietary fat composition in humans. American Journal of Clinical Nutrition 76:1222-1229

2. Anzalone $\mathrm{A}$, Carbuhn $\mathrm{A}$, Jones $\mathrm{L}$ et al. (2019) The Omega-3 Index in National Collegiate Athletic Association Division I Collegiate Football Athletes. Journal of Athletic Training 54 (1):7-11

3. Blue MN, Trexler ET, Hirsch KR et al. (2019 ) A profile of body composition, omega-3 and Vitamin D in National Football League players. Journal of Sports Medicine and Physical Fitness:87-93

4. Burke LM (2001) Nutritional Practices of Male and Female Endurance Cyclists. Sports Medicine 31 (7):521-532

5. Calder PC, Lindley MR, Burke LM et al. (2010) A-Z of nutritional supplements: dietary supplements, sports nutrition foods and ergogenic aids for health and performance Part 14. British Journal of Sports Medicine 44 (14):1065-1067

6. Davinelli S, Corbi G, Righetti $S$ et al. (2019) Relationship Between Distance Run Per Week, Omega-3 Index, and Arachidonic

Acid (AA)/Eicosapentaenoic Acid (EPA) Ratio: An Observational Retrospective Study in Non-elite Runners. Front Physiol 10:487

7. Drobnic F, Rueda F, Pons V et al. (2017) Erythrocyte Omega-3 Fatty Acid Content in Elite Athletes in Response to Omega-3 Supplementation: A Dose-Response Pilot Study. Journal of Lipids 2017:1-7

8. Fernández-García B, Pérez-Landaluce J, Rodríguez-Alonso $\mathrm{M}$ et al. (2000) Intensity of exercise during road race pro-cycling competition. Medicine and Science in Sport and Exercise 35 (5):10021006

9. Harris W, Von Schacky C (2004) The Omega-3 Index: a new risk factor for 
death from coronary heart disease? Preventative Medicine 39:212-220

10. Harris WS, Polreis J (2016) Measurement of the Omega-3 Index in Dried Blood Spots. Annals of Clinical and Laboratory Research 04 (04)

11. Helge JW, Wu BJ, Willer $\mathrm{M}$ et al. (2001) Training affects muscle phospholipid fatty acid compostion in humans. Journal of Applied Physiology 90:670-677

12. Hingley L, Macartney MJ, Brown MA et al. (2017) DHA-rich Fish Oil Increases the Omega-3 Index and Lowers the Oxygen Cost of Physiologically Stressful Cycling in Trained Individuals. International Journal of Sport Nutrition and Exercise Metabolism 27 (4):335-343

13. Kerksick CM, Arent S, Schoenfeld BJ et al. (2017) International society of sports nutrition position stand: nutrient timing. Journal of the International Society of Sports Nutrition 14:33

14. Macartney M, Hingley L, Brown M et al. (2014) Intrinsic heart rate recovery after dynamic exercise is improved with increased omega-3 index in healthy males. British Journal of Nutrition 112:1984-1992

15. Macartney MJ, Peoples GE, McLennan PL (2020) Cardiac Arrhythmia Prevention in Ischemia and Reperfusion by Low-Dose Dietary Fish Oil Supplementation in Rats. Journal of Nutrition 150 (12):3086-3093

16. Macartney MJ, Peoples GE, Treweek TM et al. (2019) Docosahexaenoic acid varies in rat skeletal muscle membranes according to fibre type and provision of dietary fish oil. Prostaglandins Leukotrienes and Essential Fatty Acids 151:37-44

17. McLennan PL (2014) Cardiac physiology and clinical efficacy of dietary fish oil clarified through cellular mechanisms of omega-3 polyunsaturated fatty acids. European Journal Applied Physiology 114 (7):1333-1356

18. Micha $\mathrm{R}$, Khatibzadeh $\mathrm{S}$, Shi $\mathrm{P}$ et al. (2014) Global, regional, and national consumption levels of dietary fats and oils in 1990 and 2010: a systematic analysis including 266 country-specific nutrition surveys. BMJ: British Medical Journal 348:g2272

19. Novak AR, Dascombe BJ (2014) Physiological and performance characteristics of road, mountain bike and BMX cyclists. Journl of Science and Cycling 3 (3):9-16

20. Peoples G, McLennan P, Howe P et al. (2008) Fish oil reduces heart rate and oxygen consumption during exercise Journal of Cardiovascular Pharmacology 52:540-547

21. Ritz PP, Rogers MB, Zabinsky JS et al. (2020) Dietary and Biological Assessment of the Omega-3 Status of Collegiate Athletes: A Cross-Sectional Analysis. PLoS ONE 15 (4):e0228834

22. Santalla A, Earnest CP, Marroyo JA et al. (2012) The Tour de France: An Updated Physiological Review. International Journal of Sports Physiology and Performance 7:200-209

23. Simopoulos AP (2007) Omega-3 fatty acids and athletics. Current Sports Medicine Reports 6 (4):230-236

24. Slee EL, McLennan PL, Owen AJ et al. (2010) Low dietary fish-oil threshold for myocardial membrane n-3 PUFA enrichment independent of n-6 PUFA intake in rats. Journal of Lipid Research $\mathbf{5 1}$ (7):1841-1848

25. Stark KD, Van Elswyk ME, Higgins MR et al. (2016) Global survey of the omega3 fatty acids, docosahexaenoic acid and eicosapentaenoic acid in the blood stream of healthy adults. Progress in Lipid Research 63:132-152

26. von Schacky C, Kemper M, Haslbauer R et al. (2014) Low Omega-3 Index in 106 German Elite Winter Endurance Athletes: A Pilot Study. International Journal of Sport Nutrition and Exercise Metabolism 24: 559 -564 


\section{Annexes}

Table 2. Whole blood fatty acid profile (\%) at baseline, before and after the TdF.

\begin{tabular}{|c|c|c|c|c|c|c|c|c|c|}
\hline \multirow{2}{*}{$\begin{array}{c}\text { Fatty acid } \\
(\%)\end{array}$} & \multicolumn{3}{|c|}{ Rider 1} & \multicolumn{3}{|c|}{ Rider 2} & \multicolumn{3}{|c|}{ Rider 3} \\
\hline & Baseline & Pre- & Post- & Baseline & Pre- & Post- & Baseline & Pre- & Post- \\
\hline $14: 0$ & 1.42 & 0.70 & 0.92 & 0.52 & 0.64 & 0.98 & 0.35 & 0.66 & 0.71 \\
\hline $16: 0$ & 21.63 & 22.12 & 21.88 & 21.68 & 22.67 & 21.80 & 20.30 & 22.85 & 21.75 \\
\hline C18:0 & 10.83 & 12.02 & 11.01 & 11.19 & 11.66 & 10.89 & 11.17 & 11.79 & 10.92 \\
\hline C20:0 & 0.34 & 0.27 & 0.28 & 0.20 & 0.21 & 0.25 & 0.27 & 0.27 & 0.31 \\
\hline C22:0 & 1.11 & 0.69 & 0.80 & 0.69 & 0.48 & 0.74 & 0.96 & 0.72 & 0.68 \\
\hline C24:0 & 1.69 & 1.23 & 1.33 & 1.37 & 0.88 & 1.24 & 1.80 & 1.27 & 1.10 \\
\hline Sum of SFA & 37.02 & 37.03 & 36.22 & 35.64 & 36.54 & 35.89 & 35.85 & 37.57 & 35.48 \\
\hline C16:1n7t & 0.12 & 0.09 & 0.15 & 0.09 & 0.10 & 0.11 & 0.06 & 0.05 & 0.08 \\
\hline C16:1n7 & 0.83 & 0.71 & 0.71 & 0.76 & 0.84 & 0.74 & 0.77 & 1.00 & 0.62 \\
\hline C18:1t & 0.25 & 0.26 & 0.47 & 0.32 & 0.33 & 0.56 & 0.28 & 0.26 & 0.29 \\
\hline C18:1n9 & 23.09 & 17.64 & 18.77 & 20.22 & 18.40 & 19.03 & 20.39 & 18.60 & 19.66 \\
\hline C20:1n9 & 0.21 & 0.19 & 0.26 & 0.19 & 0.16 & 0.27 & 0.18 & 0.19 & 0.28 \\
\hline C24:1n9 & 1.63 & 1.67 & 1.74 & 1.53 & 1.09 & 1.71 & 2.00 & 1.65 & 1.71 \\
\hline $\begin{array}{l}\text { Sum of } \\
\text { MUFA }\end{array}$ & 26.06 & 20.55 & 22.09 & 23.11 & 20.90 & 22.41 & 23.68 & 21.75 & 22.64 \\
\hline C18:2n6t & 0.22 & 0.15 & 0.25 & 0.20 & 0.16 & 0.16 & 0.13 & 0.14 & 0.14 \\
\hline C18:2n6 & 19.24 & 21.71 & 20.30 & 22.38 & 22.24 & 21.16 & 19.62 & 19.77 & 21.23 \\
\hline C18:3n6 & 0.25 & 0.20 & 0.14 & 0.11 & 0.14 & 0.12 & 0.11 & 0.24 & 0.12 \\
\hline C20:2n6 & 0.20 & 0.23 & 0.26 & 0.69 & 0.19 & 0.27 & 0.22 & 0.19 & 0.29 \\
\hline C20:3n6 & 1.21 & 1.17 & 1.22 & 1.01 & 0.86 & 1.15 & 0.77 & 0.96 & 1.07 \\
\hline C20:4n6 & 8.94 & 8.67 & 8.59 & 9.78 & 7.91 & 7.84 & 9.22 & 7.49 & 6.96 \\
\hline C22:4n6 & 0.91 & 0.92 & 0.80 & 0.90 & 0.77 & 0.64 & 1.45 & 1.15 & 0.96 \\
\hline C22:5n6 & 0.36 & 0.33 & 0.36 & 0.45 & 0.27 & 0.36 & 0.61 & 0.42 & 0.43 \\
\hline $\begin{array}{c}\text { Sum of } \\
\text { omega-6 } \\
\text { PUFA }\end{array}$ & 31.33 & 33.39 & 31.92 & 35.00 & 32.54 & 31.70 & 32.12 & 30.41 & 31.20 \\
\hline C18:3n3 & 0.22 & 0.15 & 0.27 & 0.20 & 0.20 & 0.27 & 0.29 & 0.22 & 0.43 \\
\hline C20:5n3 & 1.09 & 2.01 & 2.38 & 0.76 & 3.32 & 2.93 & 1.80 & 1.65 & 1.92 \\
\hline C22:5n3 & 1.05 & 2.66 & 2.92 & 1.27 & 2.47 & 2.66 & 1.74 & 3.10 & 3.05 \\
\hline C22:6n3 & 3.22 & 4.21 & 4.19 & 4.02 & 4.12 & 4.13 & 5.52 & 5.30 & 5.28 \\
\hline $\begin{array}{c}\text { Sum of } \\
\text { omega-3 } \\
\text { PUFA }\end{array}$ & 5.59 & 9.03 & 9.76 & 6.25 & 10.02 & 9.99 & 9.35 & 10.27 & 10.68 \\
\hline
\end{tabular}

